\title{
«La responsabilidad social nace de una voca- ción, de algo que es libre por naturaleza» Entrevista a Patricia Ruiz Bravo, directora de la Dirección Académica de Responsabilidad Social, a cargo de Alfredo Villavicencio
}

\section{Alfredo Villavicencio: A casi un siglo de existencia de la Universi- dad Católica, icómo podrías graficarnos la relevancia del tema de la proyección social y la responsabilidad social en el quehacer de la universidad?}

Patricia Ruiz Bravo: Desde la fundación de la universidad, la preocupación por la proyección social y por la responsabilidad social ha sido constante. Desde esos años iniciales, la universidad ha desarrollado una línea de reflexión y práctica sobre las relaciones que se deben establecer con la comunidad a partir tanto de una preocupación por el prójimo como por una solidaridad con los pobres y una demanda por la justicia social. Por ello, desde sus inicios, la impronta católica de la universidad está ligada a este vínculo con los otros, con aquellos que no pueden acceder a esta casa de estudios pero con los cuales tenemos un compromiso vital. Se trata de promover un trabajo con esos otros que no tienen acceso a esta educación pero tampoco a la justicia y la igualdad. Esta reflexión es parte de la formación integral que la universidad plantea y por ello primero la proyección social y luego la responsabilidad social universitaria son ejes centrales de la propuesta educativa de la PUCP.

Ahora bien, con los años, la manera de entender este vínculo y las estrategias de acción que es necesario adoptar han ido cambiando. Pero lo más importante en esta primera aproximación es que desde su formación la universidad ha pensado y se ha preocupado por este lazo: la universidad tiene que estar vinculada a la sociedad de la que es parte. En los años iniciales, se veía a la universidad más desde el lado del saber que se proyecta a quienes carecen de él. Era una mirada de un tinte más paternalista, asistencial y de servicio, pero que aún no reconocía esas otras formas de saber. La diversidad es parte de un contexto y hay que mantener una mirada histórica que permita comprender las diferentes concepciones y estrategias de acción. Actualmente, la proyección social ya no se ve solamente desde este enfoque paternalista, sino también como un diálogo a través del cual se trata de establecer una relación entre iguales. La universidad tiene la función de colaborar con el desarrollo de la sociedad y debe poner su saber y su conocimiento al servicio de la sociedad.

¿Consideras que esta posición se ve reflejada en lo que dentro de la universidad inicialmente se llamó «proyección social» y hoy se llama «responsabilidad social»? 
Yo creo que sí, porque cuando se forma la Dirección de Proyección Social, que ahora se llama Dirección Académica de Responsabilidad Social (DARS), ya el enfoque es más de trabajo con las comunidades, de un diálogo de saberes. La diferencia básica que yo encuentro con la Dirección de Responsabilidad Social es que el enfoque de responsabilidad social tiene dos énfasis distintos, no es que no hayan estado presentes en el tema de proyección social, sino que ahora son más explícitos y se llama más la atención sobre ellos.

El primero, se refiere a la manera en que se entiende la relación entre la universidad y su entorno. Ahora se enfatiza más que antes una relación de ida y vuelta, que implica no solo un reconocimiento de otros saberes — distintos del académico — sino que reconoce también la importancia de que estas otras formas de entender y de conocer ingresen a la universidad y renueven su quehacer.

Entonces, el vínculo que debe tener la universidad no sería una flecha que va desde los centros académicos hacia fuera, sino que esto regresa a la universidad planteándonos retos y preguntas tales como iqué demanda la sociedad actual a la Universidad Católica? ¿Qué cosas debemos enseñar e investigar? ¿Cómo debemos formar a nuestros estudiantes en un país caracterizado por 35\% de pobreza, con identidad multicultural, con discriminación, y con gente que es emprendedora, con espíritu luchador que sabe enfrentarse a las dificultades? Entonces, con esta sociedad, iqué nuevos retos tiene la universidad? Ese es el primer aspecto: icomo respondemos a esas nuevas demandas de la sociedad?

Pero también existe otra dimensión que es importante desarrollar: ide qué manera la universidad, en este vínculo con comunidades pobres, que están en situación de vulneración de sus derechos, recoge los aprendizajes y promueve cambios en la docencia, en la investigación, en las mallas curriculares? En el enfoque de responsabilidad social el concepto de diálogo de saberes es central, pues orienta estratégicamente las acciones a desarrollar.

\section{¿En qué sentido?}

Por ejemplo, uno puede estar en una comunidad dictando una capacitación sobre formas de cultivo de ciertos productos, pero debemos partir de reconocer que esta población tiene también ciertos saberes sobre los cultivos, sobre la salud, sobre el Derecho, sobre cómo se organizan socialmente. Dichos saberes tienen que entablar un diálogo con lo que nosotros enseñamos, puesto que aquello que les enseñamos está vinculado con una tradición occidental que no ha conversado con las tradiciones peruanas ni con nuestras tradiciones sociales. Entonces no se trata, pienso, de decir que todo se tiene que cambiar, sino de hacer hincapié en el efecto que este diálogo de saberes tiene en la universidad. 
Existe una interacción, entonces, entre la universidad y la sociedad, y la sociedad y la universidad, que busca promover cambios o resolver problemas en una dirección y en otra, iverdad?

Así es, porque de otra manera la proyección social se vería unidireccionalmente y solo se reconocería a la universidad como difusora de conocimientos y promotora de cambios, y ello representaría olvidar vernos a nosotros mismos como objeto de cambio. Creo que esto es muy importante: la capacidad de autoevaluación, la disposición al cambio. De lo contrario, no hay autocrítica.

Y creo que la responsabilidad social puede verse como esa piedra en el zapato que es como una conciencia crítica. Es difícil pensar en una renovación creativa de nuestro quehacer. La responsabilidad social supone no solamente gestión sino que — tal y como lo entiende el equipo rectoral actual- es parte de la misión de la universidad. En los foros y encuentros interuniversitarios se habla de responsabilidad social como la tercera misión de las universidades, pues ella implica ponerse a reflexionar sobre la pertinencia de nuestro quehacer, sobre las maneras de enseñar, sobre los vínculos con el entorno y sobre la propia transformación de la universidad en un contexto de cambios.

Ahora, un último tema que ha entrado en el enfoque de responsabilidad social tiene que ver con la declaración de UNESCO del año 2005, que señala que en este proceso de cambios y de globalización se plantean problemas para un desarrollo sostenible frente al cual la universidad socialmente responsable - tiene que responder. Por ello, las instituciones de educación superior deben preocuparse tanto desde la docencia como desde la investigación, la gestión y la organización de estos temas. Esto abre otra línea de acción, nuevamente hacia adentro y hacia fuera. Hacia adentro, el objetivo es caminar hacia un campus sostenible, lo cual va a suponer identificar problemas y elaborar propuestas de cambio. Por ejemplo, en coordinación con Clima de Cambios, vamos a iniciar una campaña para mejorar el manejo de los residuos e impulsar nuevas prácticas en el consumo de plásticos no renovables y que son dañinos a la salud, que esperamos introduzcan cambios en las prácticas y en las actitudes.

\section{Cuéntanos más sobre esta interesante campaña.}

Clima de Cambios es una iniciativa de la universidad que trata de poner el foco sobre el problema del cambio climático y del desarrollo sostenible. Esta campaña, que no es una propuesta coyuntural, sino una propuesta de política de la universidad, supone posicionarnos como una universidad que se está preocupando por un tema que es relevante nacional e internacionalmente. Su propósito es empezar a tomar conciencia, a sensibilizar y a tomar acciones correctivas tanto en el interior como en el exterior de la universidad. 
Clima de Cambios acoge y busca apoyar todas las iniciativas de las distintas unidades. Así, por ejemplo, si una facultad, departamento o centro de investigación quisiera promover cambios en las mallas curriculares o poner en marcha programas de investigación o de desarrollo, puede coordinar con Clima de Cambios, ahora alojada en la Dirección de Comunicación Institucional, para acogerse a su paraguas. De esta manera, y al ubicarse en este paraguas que Clima de Cambios representa, las actividades y propuestas que la universidad desarrolla de manera creciente pueden estar más articuladas y ser mejor difundidas en el medio

\section{¿Es un eje transversal, entonces, en la actividad de la universidad?}

En efecto, es un eje transversal y es parte de la misión de la dirección que tengo a mi cargo. Ello supone trabajar en varios frentes: con docentes y estudiantes, pero también con personal administrativo, pues muchas prácticas se deben cambiar en estos tres estamentos. Entonces, nuestro trabajo actual es acercarse a facultades, departamentos, organizaciones de estudiantes y direcciones para conversar y estimular el desarrollo de iniciativas en estas líneas de trabajo. Algunas unidades hacen un excelente trabajo de responsabilidad social, pero aún hay mucho por trabajar. Nuestra estrategia es apoyarnos y apoyar las experiencias ya existentes, que son conocidas, para motivar a otras unidades a que realicen actividades de este tipo.

Si ahora viésemos dos ejes, primero, icuáles son las políticas institucionales de la universidad?, y luego, icuáles son las experiencias que se están desarrollando a nivel de las unidades? En el primer caso, además de Clima de Cambios, iqué otras políticas institucionales está llevando a cabo la universidad en cuanto a responsabilidad social?

Bueno, aparte de Clima de Cambios, una política clave fue la creación de esta dirección. Se asumió que ella tomaría decisiones que luego se transformarían en propuestas de políticas. Yo he sido elegida para este cargo en agosto de 2009 y se espera que, para fines de este año, podamos presentar al rectorado propuestas de políticas de lo que sería responsabilidad social para nosotros. Por ejemplo, durante Consejo Universitario se aprobó una política que admite que el reconocimiento para los profesores involucrados en responsabilidad social no solamente debe ser subjetivo y moral, sino también económico. La universidad señala que el profesor debe enseñar, investigar y practicar responsabilidad social. Entonces, ipor qué los premios y los aumentos solo guardan relación con docencia e investigación? Tras ser discutido, se aprobó que en 2010 se entregará un bono como reconocimiento para los profesores que hayan destacado al llevar a cabo proyectos y actividades de responsabilidad social. ¿Por qué me parece importante este premio? Porque uno no puede admitir una política que reconozca la importancia de la responsabilidad social sin incluir premios por ella. 
No hay muchos fondos, pero la idea es que la universidad conozca y reconozca este trabajo. Varios docentes vienen impulsando actividades sobre esta línea de trabajo, pero su labor no es conocida por sus pares de otras disciplinas. Así se pierden posibilidades de trabajo interdisciplinario de mucho mayor impacto Los más conocidos y cuya trayectoria es importante son los docentes y estudiantes de Derecho, por todo el trabajo que viene haciendo con PROSODE, que creo que ya tiene más de veinte años. En Arte y en Psicología también existen grupos muy comprometidos con la responsabilidad social.

\section{¿Qué hace Psicología en cuanto a responsabilidad social?}

Es bien interesante, porque Psicología tiene una unidad de responsabilidad social directiva que no se ha llegado a cumplir en todos los departamentos: ellos planifican acciones para el año. Entre sus líneas de trabajo se cuentan la labor en penales, el apoyo a comunidades cristianas y las brigadas psicológicas, que se ocupan del tema de salud mental comunitaria. Sucede que muchas poblaciones no acceden a los servicios psicológicos, y hay que diseñar propuestas para que la psicología esté al servicio de todos, no solo para quienes tengan recursos. Estas brigadas, que inicialmente han trabajado después de los terremotos, se están repensando para otras situaciones de emergencia y crisis.

\section{En cuanto a las líneas de trabajo de PROSODE, $i$ cuál identificas como más relevante?}

Creo que PROSODE es un ejemplo para la universidad al ser una iniciativa de la propia unidad, gracias al interés y al compromiso de docentes y estudiantes, quienes, de manera sistemática, se han preocupado por desarrollar proyectos. En cuanto a sus líneas de trabajo, creo que cada una tiene su razón de ser. Los consultorios jurídicos en zonas de escasos recursos me parecen claves y deben seguir funcionando. Inclusive me parece que el decano comentaba acerca de una iniciativa para hacer un consultorio para el personal de la universidad.

Alrededor de esta idea de «adentro y afuera», creo que el programa de radio se podría potenciar muchísimo más. Me parece que existe un proyecto para hacer una radio universitaria. Sería sumamente importante que estas campañas o programas radiales llegaran a concretarse porque hay que trabajar mucho con la educación. La educación constituye una de las líneas que me gustaría desarrollar en este vínculo universidad-sociedad, tal vez no con escuelas primarias, pero sí con colegios de secundaria para desarrollar temas de formación cívica y moral, y análisis de la realidad social. Pienso que, por ejemplo, los conocimientos de psicología comunitaria deberían entrar en las mallas curriculares de la educación secundaria. 
¿La universidad no cuenta con programas que busquen sensibilizar, sobre estas líneas, a profesores de secundaria o de primaria?

Ha habido iniciativas de varios docentes y de algunas facultades, pero aún no suponen una política. En ello me gustaría trabajar durante estos años. Hemos visto propuestas muy innovadoras que nos interesa impulsar y desarrollar. Por ejemplo, el profesor Jesús Cosamalón, de Historia, ha desarrollado un blog para profesores escolares. Ahí «cuelga» documentos para que preparen mejor sus clases o para que accedan a nuevos enfoques, y también responde a las preguntas que ellos plantean. Así promueve un diálogo enriquecedor para ambas partes. Trabaja con un grupo de estudiantes y todos se reúnen con docentes de colegios. Así impulsa un trabajo colectivo.

Esta preocupación por la Historia y por la manera en que se enseña ha generado un proyecto de rectorado que trata de ir promoviendo una reflexión sobre la celebración del Bicentenario de la Independencia. A lo largo de este camino - porque todavía nos falta un tiempo-, se reflexionará sobre el significado de la Independencia hoy. Somos libres e independientes en un sentido, sí, pero en muchos otros no. La idea es ir trabajando con una propuesta ya no para los grandes expertos sino para las escuelas. La idea es ir desarrollando una reflexión sobre estos temas e ir generando debates e irradiar a una comunidad más amplia.

El Bicentenario de la Independencia es una ocasión para repensar nuestro pasado y mirar nuestro futuro. Esperamos contribuir a una reflexión sobre el país y la ciudadanía. Regularmente, cuando se piensa en la Independencia, solo se trabajan las figuras de los próceres, San Martín y de Bolívar, y se olvidan el proceso y el ejercicio permanente de la libertad. Entonces, llegar al bicentenario implica formar esta conciencia ciudadana pero desde un lenguaje que llegue a todos. Para ello la universidad está elaborando este programa, y vamos a empezar a trabajar con dos o tres colegios de Pueblo Libre y con familias que son de nuestro entorno inmediato.

\section{¿Qué otras iniciativas habría que poner en relieve? ¿En qué estás abocada ahora, fuera de las tres que ya has comentado?}

Habría que destacar que esta universidad está llena de iniciativas. Tal vez mi misión, en un primer momento, sea hacerlas conocidas y potenciarlas. Hay un trabajo muy interesante en Arte. Un grupo que tiene ya muchos años de experiencia, Axis Arte, trabaja con artesanos de la costa norte del Perú; en particular, conozco su trabajo en Túcume. Primero conducen una investigación sobre la iconografía de la zona: cuál es el significado de sus íconos, cómo se asocian a la vida comunitaria, cuáles son usados y de qué manera. Después, capacitan a los artesanos y a las personas vinculadas con la producción de estos artefactos, que en algunos casos son telares u objetos utilitarios. El propósito es que mejoren sus 
diseños, pero también que la recuperación de su historia sea incorporada al currículo escolar.

Su proyecto tiene dos ejes: uno económico, pues se trata de que los artesanos mejoren sus ingresos; y otro cultural, pues se trata de un positivo reconocimiento de su identidad. Esto último se hace a través de la escuela, de un programa en la malla curricular, siguiendo las demandas ministeriales de diversificación curricular. Han apoyado, además, el desarrollo de contenidos al publicar materiales educativos y textos escolares.

Otra experiencia interesante es la de la Facultad de Ciencias e Ingeniería. He tenido contacto, en estos últimos meses, con el Grupo de Apoyo al Sector Rural, el cual ha desarrollado importantes propuestas para mejorar la calidad de vida de poblaciones andinas. Sus integrantes han conducido investigaciones e intervenciones en zonas de altitud, y han desarrollado tecnologías ad hoc que están dando resultados interesantes. Es el caso de las cocinas mejoradas y del «muro trombe», que posee ciertas características para aislar mejor el frío. Este proyecto se está llevando ahora en Cusco. Regresa a los estudiantes como un estímulo, pues se trata de pensar en cómo podemos, desde la universidad, innovar para colaborar con el mejoramiento de la calidad de vida, en este caso de nuestros hermanos de las zonas altoandinas.

Otro trabajo muy interesante fue la investigación sobre el adobe y la manera de hacer que las construcciones de adobe, mayoritarias en las zonas rurales y de menores recursos, puedan ser seguras y antisísmicas. Un grupo de ingenieros ha investigado este tema por muchos años y ha desarrollado varias propuestas para la construcción con adobe reforzado. Por el trabajo en la DARS he tenido más contacto con la propuesta del adobe reforzado con geomalla, que es el modelo que se ha seguido para la reconstrucción de algunas casas en las zonas afectadas por el sismo de 2007. Esta propuesta, desarrollada por los ingenieros Blondet, Torrealva y Vargas, ya ha sido aprobada por el Ministerio de Vivienda, ha sido asumida y va a ser parte de una política para la vivienda rural. Este es un caso de una investigación de alto impacto social y que ha permitido impulsar una política de estado para desarrollar viviendas rurales.

Luego, otro investigador — porque, como te digo, la responsabilidad social tiene que ver con docencia, investigación y proyectos- está realizando estudios sobre incubadoras que puedan servir en zonas de altitud. El mencionado profesor desarrolló esta idea como respuesta a una necesidad para nuestro país, y eso es lo que nosotros remarcamos como parte fundamental de un profesor de la universidad: que alguien que hace bien sus cosas puede hacer tecnología de punta, que esté conectado a la tecnología necesaria para nuestra sociedad.

En Física, y en convenio con un docente francés, se está trabajando en un bastón para ciegos que funciona electrónicamente y que va a mejorar la calidad de vida de personas discapacitadas. Ahora bien, en este caso 
el camino es largo, pues se necesita capacitar a los ciegos que puedan ser los usuarios de esta nueva tecnología. Para ello se busca firmar un convenio con un instituto que pueda hacerse cargo de esta tarea. Otro ejemplo lo tenemos en Ingeniería Mecánica, donde se están diseñando unas máquinas para reciclar botellas de plástico y se está firmando un convenio con la institución EDUCA, que apoya a recicladores, para que canalice estas iniciativas.

Para cerrar, como conductora de la política institucional, icuáles son tus grandes metas? ¿Cómo ves los resultados, el trabajo, la sistematización de la DARS, si trataras de avizorar el fin de tu mandato en esta primera edición?

Creo que me gustaría llegar a desarrollar políticas de responsabilidad social que tengan que ver tanto con docencia como con investigación y manejo sostenible del campus, en varios sentidos. Mi imagen ideal es que, cuando termine mi mandato, cada unidad ya tenga su espacio, su propio órgano de responsabilidad social, y entonces mi oficina siga funcionando simplemente como una oficina de coordinación y dirección. Porque como dirección académica tengo un espacio en el Consejo Universitario, y eso es clave para la atención y para la implementación de políticas. Pienso que es necesario ser parte de un órgano de gobierno para vigilar el cumplimiento de dichas políticas. Es clave definir sus líneas, que pasan por institucionalizar el compromiso y la vocación de responsabilidad social en las unidades.

Y aquí encuentro una tensión que es importante debatir y manejar, pues no es de fácil solución: la responsabilidad social nace de una vocación, de algo que es — vamos a decirlo así - libre por naturaleza, y por ello a la gente que se compromete con ella no le gusta estar bajo parámetros, bajo normas. Por eso el voluntariado es tan interesante: los estudiantes son voluntarios pero no quieren que el voluntariado se institucionalice, pues se corre el riesgo de hacerlo burocrático, de quitarle la frescura que debe tener. Es esta, pues, la tensión en la que nos debemos mover: de un lado, dar espacio a las diversas iniciativas y a la creatividad; pero, a la vez, sistematizar y desarrollar líneas institucionales que, sin ahogar ni aprisionar tales iniciativas, puedan asegurar su continuidad y su mejora. Para ello es muy importante vincularnos más estrechamente a las facultades, a los departamentos, a los centros federados, y hacer un trabajo conjunto, desarrollar propuestas que vengan de ellos y que yo pueda canalizar hacia el rectorado. 